\title{
Segmentation of 3D Stereoscopic Images Using LoG Operator for the Detection of Excessive Disparities
}

\author{
Sang Hyun Kim, Jeong Yeop Kim, and Gil Ja So, Member, IACSIT
}

\begin{abstract}
In the 3D stereoscopic images, there are very important that viewer does not feel the discomfort and fatigue. In order to detect the discomfort and fatigue in 3D stereoscopic images, the proposed method detects the excessive disparity regions using the adaptive LoG operator and binarization. The proposed method makes binary image of disparity-map using histogram-based binarization. It also performs region segmentation of the disparity-map using the adaptive LoG operator. In the segmented regions, the overlapped regions with the binarization results are detected as the excessive disparity regions.
\end{abstract}

Index Terms-3D stereoscopic images, binarization, LoG operator, discomfort and fatigue, disparity-map.

\section{INTRODUCTION}

Recently, the demand for 3D stereoscopic images is growing sharply. Typically, the real 3D stereoscopic image contents are made by bilateral approach using two cameras, in other words, each side of the camera to acquire images at the same time. 3D stereoscopic image using two cameras are inputted left and right eyes. Viewer synthesizes the left and right images and feels depth in his brain. This approach should ensure uniformity of left and right images to provide the safe and comfortable viewing [1], [2].

The conventional stereo matching estimates the relation between corresponding pairs, calculates disparity and depth information. It is the method [3]-[5] to describe 3D surface to acquire depth information for all pixels in the image using interpolation.

The viewer discomfort issue is especially important for children as their vision systems have not grown mature and their inter-pupil distances are shorter than adults. It is need to prevent that the children will not be exposed to excessive disparities and thus have no additional eye fatigue due to $3 \mathrm{D}$ stereoscopic image [6].

In [6], the discomfort and fatigue in 3D stereoscopic images occur from the recognition error of depth information made by excessive positive and negative disparities. These the recognition error of depth information is detected by measurement of perceived depth on screen and fixed by depth tuning methods as the depth shift and the depth scaling.

Manuscript received November 1, 2012; revised January 24, 2013.

S. H. Kim and G. J. So are with the Department of Computer Engineering of the University of Young-San at Yangsan, Kyungnam (e-mail: ksh50@ ysu.ac.kr,kjso@ ysu.ac.kr).

J. Y. Kim is with the Department of Game Contents Science of the University of Young-San at Busan, as an associate professor (e-mail: neocopy@ysu.ac.kr).
Here, if some perceived depths are outside the pre-defined threshold in histogram of disparity map, those perceived depths are regarded as the excessive disparities and fixed by depth tuning methods.

In detection of the excessive disparities based on the histogram, there are some problems. Because objects in 3D stereoscopic images have three-dimensional position and depth depending on the difference of lighting effects, such as smoking. The excessive disparities are also generated locally by particular objects. The histogram based method dose not correctly detects it because of inaccuracy in disparity detection made from occlusion or noise. The many small isolated excessive disparity regions may be occurred [7]-[9] To reduce the problem, it is required segmentation method additionally.

In general, image segmentation methods can be classified into image region-based methods and edge-based methods [10], [11]. LoG operator based method in edge-based methods forms closed-loop and extracts contour of each objects. It can be shown the stable segmentation results in noisy images, such as ultrasound images [12].

It is proposed the LoG operator based segmentation method [12] that adaptively segments the input image using user informed vertical size of the object and the variance of the input image.

In this paper, we propose the method detects the excessive disparity regions using the adaptive LoG operator and binarization. First it makes binary image of disparity-map using histogram-based binarization and segments disparity-map using the adaptive LoG operator. In the segmented regions, the overlapped regions with the binarization results are detected as the excessive disparity regions. In adaptive LoG operator based segmentation, we adjust the standard deviation of the Gaussian filter in LoG operator proportional to the standard deviation of disparity-map. Future, it is needed research on how to obtain the appropriate standard deviation for various images, small region elimination method, and the regression equation for the more precise adjustment of the standard deviation of the Gaussian filter.

\section{The Previous Researches}

\section{A. Yuan's Method [6]}

This method proposes the depth tuning method. The overall algorithm flow is shown in Fig. 1. The method starts by estimating $2 \mathrm{D}$ image disparities between the left and right images. The viewer's preference, e.g., input through the TV remote, is also fed into the algorithm. A human visual model 
is then applied to predict a comfortable range of image disparities.

The comfortable disparity range is compared to the image disparities as well as viewer's preference, leading to the decision of how much depth tuning is needed. Finally a view synthesis algorithm is applied to generate newer versions of the left and right images.

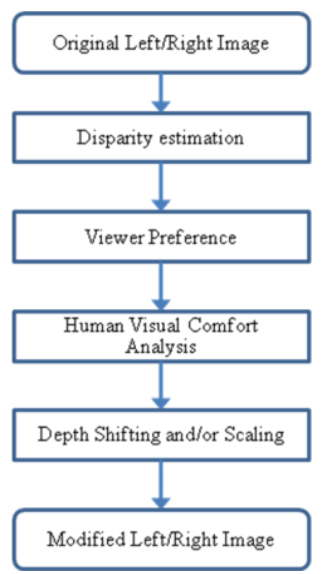

Fig. 1. Overview of the stereoscopic content tuning in Yuan's method.

Disparity estimation: A regularized block matching algorithm is used between left and right images [13].

Human visual comfort analysis: The algorithm computes the comfortable range of disparities.

Depth Tuning: The first depth tuning method, namely "depth shifting," applies lateral shifting to the two images. The second depth tuning method, "depth scaling", produces a new disparity map with scaled disparities [13].

In Fig. 2, this method's depth tuned image result from "Monsters vs Aliens 3D". This method automatically detects that the image disparities (Fig. 2 (c)) are out of the comfort zone, and applies depth scaling method.
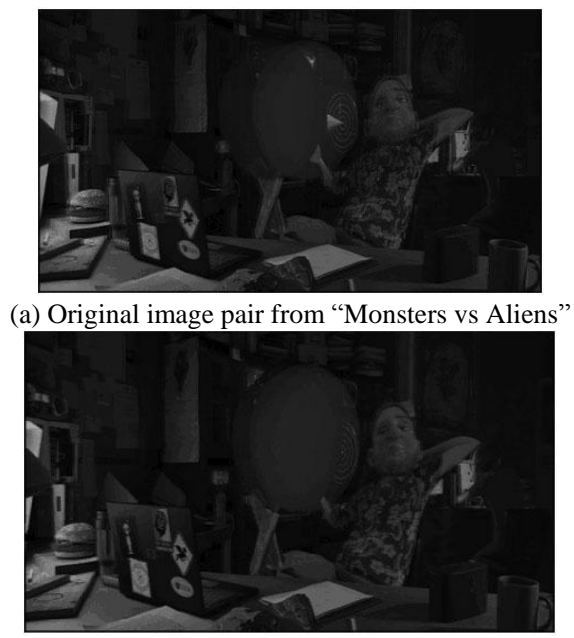

(b) Tuned image pair from "Monsters vs Aliens"

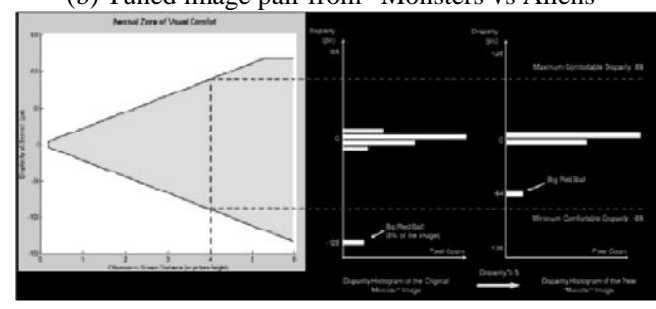

(c) Visual Comport analysis of the 3D image

Fig. 2. 3D depth tuning and analysis results on the "Monsters vs Aliens 3D" image [6].

\section{B. Contour Extraction Using LoG Operator [6]}

Contour extraction using LoG operator is shown in Fig. 1. In Fig.1, Double-differential $\nabla^{2} G$ is applied to the input image. The zero-crossing point is extracted as a contour. Here, $I(m, n)$ is a input image, $E(m, n)$ is a contour image made by zero-crossing points in the double-differential image $h(m, n)$.

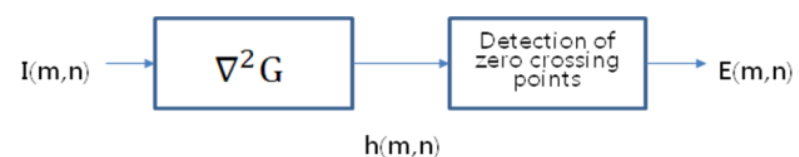

Fig. 3. Block diagram of contour extraction using LoG operator.

In Fig. 3, the equation for the procedure of contour extraction using LoG operator with the double-differential $\nabla^{2} G$ is as follows [2]:

$$
h(m, n)=\nabla^{2} G(m, n) * I(m, n)
$$

Here, LoG operator with the double-differential $\nabla^{2} G$ is as follows.

$$
\nabla^{2} G(m, n)=\frac{1}{2 \pi \sigma^{4}}\left(2-\frac{m^{2}+n^{2}}{\sigma^{2}}\right) \exp \left(-\frac{m^{2}+n^{2}}{2 \sigma^{2}}\right)
$$

If contour extraction using LoG operator is applied to the input images, then the contour images with the various resolutions, depending on the value of the standard deviation $\sigma$ can be obtained. We can obtain the variety of contour images using LoG operator by adjusting the standard deviation $\sigma$.

If the standard deviation is smaller, the effect of smoothing by the Gaussian filter is smaller. It can be obtain the detail contour image but many small regions are also occurred. In contrast, if the standard deviation is greater, the distinct contour can be detected but the objects and background can be merged. In contour extraction using LoG operator, it is important to decide the proper standard deviation.

In [6], to solve these problems, the method to automatically set the standard deviation based on the vertical position and the size informed by user, and the variance of the image, was proposed.

The 3D stereoscopic images have the disparity-map with depth information between the left and right images. The distribution in this disparity map is generated by the distribution of objects in 3D space. We can set the standard deviation proportional to the distribution as the variance in this disparity map.

\section{The Proposed METHOD}

The block diagram of the proposed method is as follows. In Fig. 4, the first the proposed method generates the disparity-map based on left image. The histogram made from the disparity-map and the standard deviation of LoG operator is decided from this. The next, region segmentation in disparity-map is performed and binariztion using threshold is 
also processed. In the segmented regions, the overlapped regions with the binarization results are detected as the excessive disparity regions.

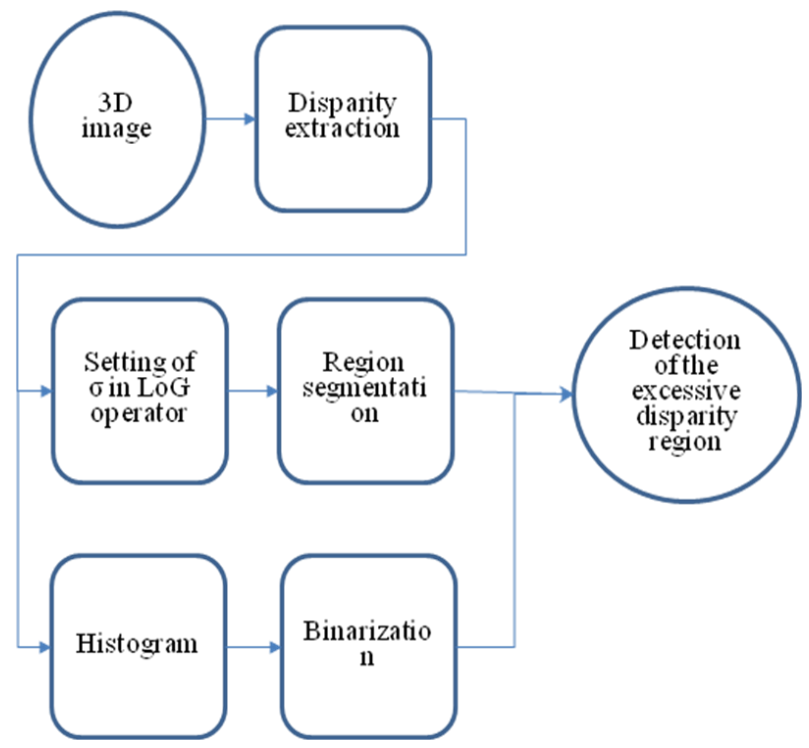

Fig. 4. Block diagram of the proposed method.

Disparity extraction: A block matching algorithm is used to calculate disparities between left and right images. In our algorithm, we cannot adopt the additive occlusion detection and processing algorithm. For simulation, mainly the ground images are used in Middlebury data set [14]. It is regarded as the correct disparity map.

Setting of standard deviation in LoG operator \& Region segmentation: The standard deviation in LoG operator is estimated using the constant of proportionality with the standard deviation of disparity-map. It is also adjusted by user preference manually. Fig. 5 shows the effect of the adjustment of standard deviation in LoG operator. If user want make the detail contour, standard deviation must be smaller. In contrast, it must be greater.

Binarization: the threshold is decided in histogram. The pixels in disparity-map, those values are over the pre-defined threshold, classified into the excessive disparity pixels.

Detection of the excessive disparity region: In the segmented regions using LoG operator, the overlapped regions with the binary results over the pre-defined threshold are detected as the excessive disparity regions.

In general, binary results have the rough boundaries and the many small regions. In the proposed method, because region segmentation using LoG operator has the smooth boundary and small region elimination function, this region segmentation using LoG operator can make up for these disadvantages occurred from binary results.

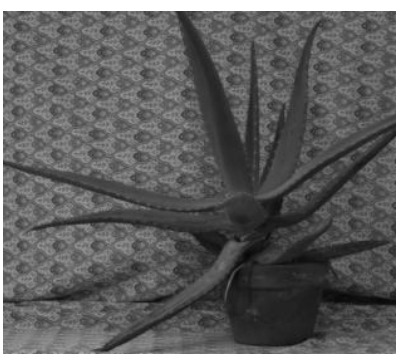

(a) Original image

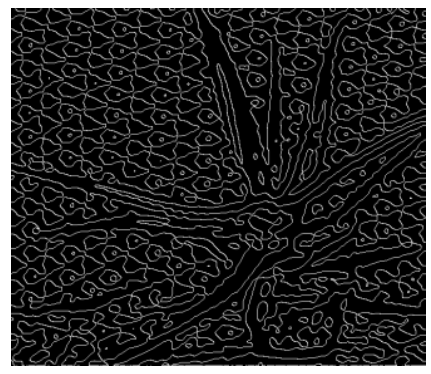

(b) Contour image obtained by LoG operator with $\sigma=5$

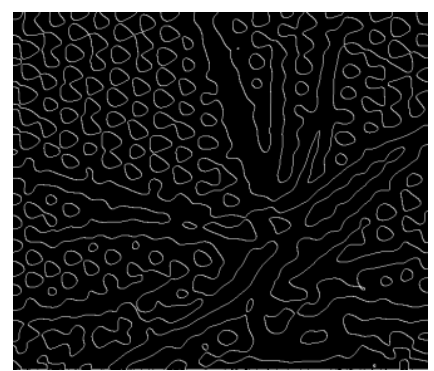

(c) Contour image obtained by LoG operator with $\sigma=10$

Fig. 5. Contour extraction results using LoG operator.

\section{Simulation Results}

Art image of 3D stereoscopic images in [14] were used in this paper's simulation. In [14], 3D stereoscopic images are composed of images with the full and half size generated by 3 types of lighting and exposure. Art original images are color images. It is converted into gray image.

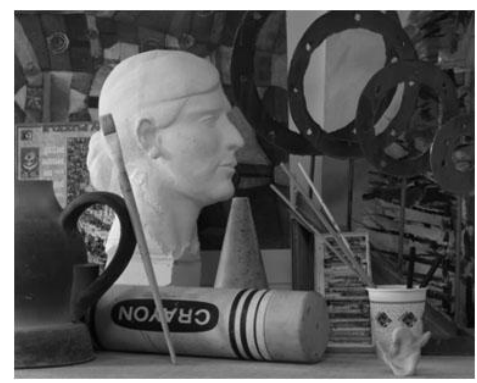

Fig. 6. Gray converted art image.

In Fig. 6, there are some objects on the table as crayon, plaster head, paper cup, and small plaster manipulation. For disparity map, we used the ground image given by Middle bury data set.

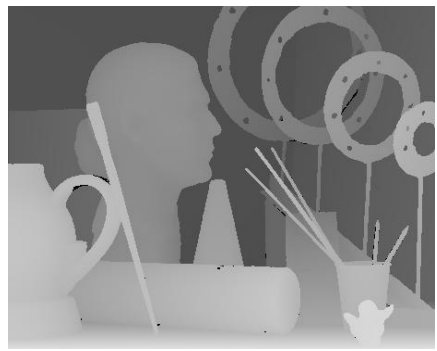

Fig. 7. Disparity-map of art image.

In Fig. 7, a small plaster manipulation has the greatest disparities. In this paper, we hope that this small plaster manipulation is extracted as the excessive disparity region. Fig.8. show the histogram of disparity-map. Disparities over the threshold=200 are marked with red circle and it is corresponded to a small plaster manipulation and additionally the front part of table. 


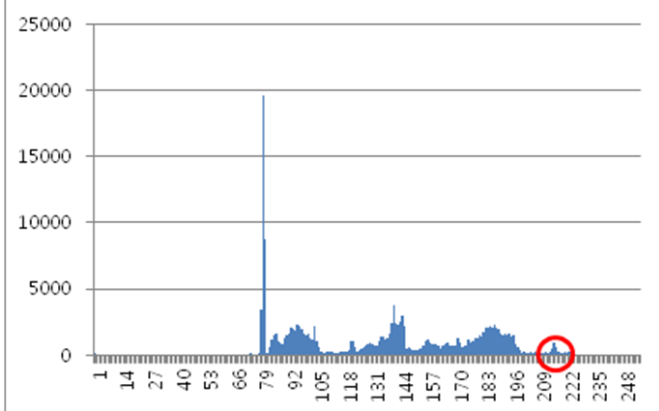

Fig. 8. Histogram of disparity-map of art image.

Fig. 9 shows the binary image and contour image. In Fig. 9, the extracted excessive disparity region, the white region, includes a small plaster manipulation and the front part of table. To adjust depth, the depth tuning algorithm can adjust the depth a small plaster manipulation but cannot adjust the front part of table. It is linked to another part. In disparity-map, it is impossible distinguish a small plaster manipulation and the front part of table have similar disparities.

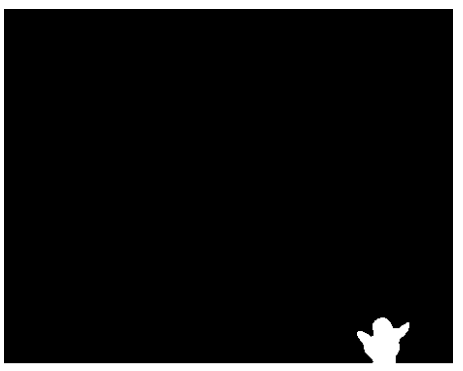

(a) Binary image

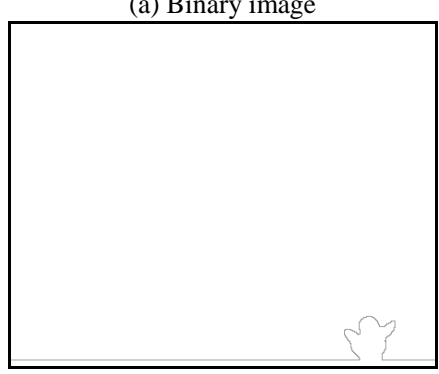

(b) Contour image

Fig. 9. Binary and contour image of the threshold result.

Therefore to distinguish objects with similar disparities and to do the effective depth tuning, in the actual 3D stereoscopic image as well as in disparity-maps, it is necessary to do segmentation and combine the results.

\section{CONCLUSION}

The viewer discomfort issue is especially important. The discomfort and fatigue in 3D stereoscopic images occur from the recognition error of depth information made by excessive positive and negative disparities.

In this paper, we propose the method detects the excessive disparity regions using the adaptive LoG operator and binarization. First it makes binary image of disparity-map using histogram-based binarization and segments disparity-map using the adaptive LoG operator. In the segmented regions, the overlapped regions with the binarization results are detected as the excessive disparity regions.

In simulation results, we extracted region with the greatest disparities as the excessive disparity region. The extracted excessive disparity regions are mixed with target and the other regions with similar disparities.

Therefore to distinguish the target objects from and the other regions with similar disparities and to do the effective depth tuning, it is necessary to do region segmentation and combine the results in the both real 3D stereoscopic left/right images and disparity-maps,.

\section{ACKNOWLEDGMENT}

This research is supported by Korea Creative Content Agency (KOCCA) in the Culture Technology (CT) Joint Research Center Project 2012.

\section{REFERENCES}

[1] C. W. Choi, " HUD factor classification of online FPS Game for 3D stereoscopic application,” M.S. thesis, Dept. of Media Broadcasting, Graduate School of Information Sciences, Soongsil Univ., Seoul, Korea, 2011

[2] S. Choi, "Technical status and prospect of 3D stereoscopic images," Journal of Korea Information Processing, vol. 17, no. 4, pp. 4-11, 2010.

[3] J. S. Kim, "A study on a balance and registration of non-rectified stereo images," Ph.D. Dissertation, Chung-Ang Univ., Seoul, Korea, 2009.

[4] S. Kim, G. So, and J. Kim, "Intensity correction of 3D stereoscopic images using region segmentation," in Proc. 34th Conf. Korea Information Processing Society, Seoul, 2010.

[5] J. S. Lee, S. D. Park, S. H. Han, and C. Y. Kim, "Implementation of 3-dimension stereoscope using 3D graphics," in Proc. Conf. Korea Multimedia Society, vol. 13, no. 2, 2010, 11, pp. 184-187.

[6] C. Yuan, H. Pan, and S. Daly, "61.3: Stereoscopic 3D content depth tuning guided by human visual models," in Proc. SID Symposium Digest of Technical Papers, vol. 42, issue 1, June 2011, pp. 916-919.

[7] Y. J. Zhang, "Improving the accuracy of direct histogram specification," Electronics Letters, vol. 28, issue. 3, pp. 213-214, 1992.

[8] H. Tuo, L. Zhang, and Y. Liu, "Multisensory aerial image registration using direct histogram specification," in Proc. IEEE Int'l Conf. Sensing and Control, vol. 2, 2004, pp. 807-812.

[9] A. Mancini, "Disparity estimation and intermediate view reconstruction for noble application in stereoscopic video," M.S. thesis, McGill University, 1998.

[10] R. C. Gonzalez and R. E. Woods, Digital Image Processing, 2nd ed. Reading, MA: Addison-Wesley, 1992.

[11] A. K. Jain, "Fundamental of digital image processing," Printice-Hall, NJ: Englewood Cliffs, 1989.

[12] M. Jung, "3D organ segmentation in ultrasound images using LoG operator," M.S. thesis, Kyung-Pook National Univ., Daegu, Korea, 2002.

[13] H. Pan, C. Yuan, and S. Daly, "3D video disparity scaling for preference and prevention of discomfort," in Proc. SPIE 7863, Stereoscopic Displays and Applications XXII, 786306, Feb. 2011.

[14] Middlebury. [Online]. Available: http://vision.middlebury.edu/vision/

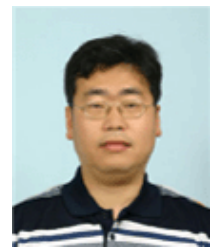

Sang Hyun Kim received the Diploma in electronics engineering from the Kyung-Pook National University of Daegu, South Korea in 1991, and MS and PhD degrees, both in image processing of electronics engineering, from the same university, in 1993 and 1998, respectively. He was with the Samsung Medison Co. Ltd, in Korea as a senior researcher from 1998 to 2001. From September 2001, he is with the Department of Computer Engineering of the University of Young-San at Yangsan, Kyungnam, as an associate professor. His doctoral dissertation work focused on the development of fractal image coding method and transmission for image processing, image communication and computer vision problems. The topics he has worked on include virtual reality, ultrasound image processing, medical image processing, image feature extraction and classification, and $3 \mathrm{D}$ stereoscopic images. His current research is on region-based intensity correction in 3D stereoscopic images. He is a member of the KMMS (Korea Multimedia Society) and KIPS (Korea Information Processing Society). 


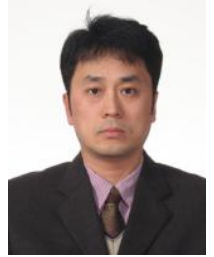

Jeong Yeop Kim received the Diploma in electronics engineering from Kyung-Pook National University of Daegu, South Korea in 1990, and MS and PhD degrees, both in image processing of electronics engineering, from the same university, in 1992 and 2000, respectively. He was with the Samsung Advanced Institute of Technology in Korea as a senior research staff from 1994 to 2000 . From

March 2001, he is with the Department of Game Contents Science of the University of Young-San at Busan, as an associate professor. His doctoral dissertation work focused on detecting and adjusting the white point from single color image. The topics he has worked on include color printing, error diffusion, color correction and 3D stereoscopic images. His current research is on correction of color data in $3 \mathrm{D}$ stereoscopic images. He is a member of the KMMS (Korea Multimedia Society) and KIPS (Korea Information Processing Society).

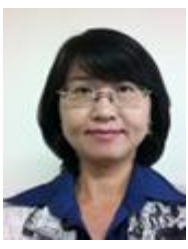

Gil Ja So received the Diploma in computer science from the Dong-Eui University of Pusan, South Korea in 1994, and MS and $\mathrm{PhD}$ degrees, both in natural language processing of computer engineering, from the Pusan National university, South Korea in 1997 and 2012, respectively. From September 2001, she is with the Department of Cyber \& Police Science of the University of Young-San at Yangsan, Kyungnam, as a lecturer. Her doctoral dissertation work focused on the Korean Grammar Checker for parsing Korean documents and correcting context-sensitive words in documents. The topics she has worked on include virtual reality, game development and artificial intelligence of non-players of game, and 3D stereoscopic images. Her current research is on region-based intensity correction in 3D stereoscopic images. She is a member of the KMMS (Korea Multimedia Society) and KIPS (Korea Information Processing Society). 